\title{
Sistematización de Experiencia en Establecimiento de Larga Estadía de Adulto Mayor: "Hacia un Enfoque Gerontológico"
}

\section{Systematizing experience in Establishment of Long Demurrage of Major Adult: " Towards an Approach Gerontological"}

\section{Verónica Véliz R. ${ }^{1}$}

\author{
Jessica Olate Andaur. ${ }^{2}$
}

\section{RESUMEN}

En base a la experiencia desarrollada con adultos mayores durante siete años en una institución de beneficencia, aplicando el enfoque psicosocial y comunitario; se observó el impacto beneficioso para la salud y funcionalidad del adulto mayor institucionalizado, al trabajar en equipo con enfoque gerontológico. Asimismo, el logro de autonomía, participación y vinculación de las personas mayores, requiere que el equipo comparta un paradigma de intervención, cuyos modelos, criterios y estrategias, faciliten el logro de objetivos terapéuticos transversales, centrados en las necesidades reales de la persona mayor y su familia.

Esta sistematización retrospectiva, de la experiencia profesional vivenciada por Enfermera y Terapeuta Ocupacional, pretende sensibilizar a profesionales y personas vinculadas con establecimientos de larga estadía, acerca de la relevancia, pertinencia y factibilidad de las intervenciones gerontológicas, que no necesariamente involucran un costo económico, más bien un cambio paradigmático en todo nivel. Para ello, se describirá el proceso realizado para la implementación de estrategias integrales y significativas, utilizando como base la conformación de equipos de diferentes disciplinas, que valoran e involucran al adulto mayor y su familia en la toma de decisiones. La comunicación y retroalimentación constante, permite mantener, mejorar o reemplazar estrategias de intervención, gracias a un proceso participativo y dinámico.

PALABRAS CLAVE: Enfoque gerontológico, sistematización de experiencia, trabajo en equipo, funcionalidad, autonomía, participación ocupacional.

\footnotetext{
${ }^{1}$ Terapeuta Ocupacional, Lic. en Cs. de la Ocupación Humana, Universidad de Chile. Corporación Desarrollo Social de San Joaquín, Dirección de Salud. veronicavelizrojas@gmail.com

${ }^{2}$ Enfermera, Licenciada en Enfermería, Universidad de Concepción. Académico Universidad de Chile, Escuela de Enfermería. jessicaolate2004@yahoo.es
} 


\section{$\underline{\text { ABSTRACT }}$}

Based on the experience developed with older adults for seven years in a charitable institution, applying the psychosocial and community approach, it was noted the beneficial impact on health and functionality of institutionalized older adults, working in teams with gerontologic approach. Also, the achievement of autonomy, participation and involvement of older people requires the team to share a paradigm of intervention, whose patterns, approaches and strategies, facilitate the achievement of cross-sectional therapeutic targets, focused on the real needs of the older person and his/her family.

This systematic retrospective of professional experience experienced by nurses and occupational therapists, aims to raise public and persons associated with long-stay facilities, about the relevance, appropriateness and feasibility of gerontological interventions, which need not involve an economic cost, more While a paradigm shift at all levels. To do so, describe the process undertaken for the implementation of comprehensive and meaningful, using as the formation of teams from different disciplines, that value and involve the elderly and their families in making decisions. Communication and constant feedback to maintain, improve or replace intervention strategies, through a participatory and dynamic process

Key Words: Gerontological approach, systematization of experience, teamwork, functionality, autonomy, occupational participation

\section{INTRODUCCIÓN}

Esta sistematización se realiza en base a la experiencia de trabajo en dos establecimientos de larga estadía de la misma institución, en diferentes periodos de tiempo. En ambas, el inicio de las intervenciones gerontológicas coincide con una reestructuración administrativa (cambio de jefatura y parte del equipo profesional), la que permite el replanteamiento respecto al impacto en la funcionalidad y calidad de vida de los adultos mayores, de las intervenciones con orientación geriátrica biomédica, implementadas hasta el momento y que se encontraban fuertemente arraigadas en el equipo de trato directo (cuidadores y paramédicos).

Al realizar el diagnóstico de situación, surgen cuestionamientos o interrogantes al observar la generalización de las medidas de tratamiento farmacológicas, cognitivas, conductuales y psicosociales, sin individualizar la atención por estado de salud, nivel cognitivo, afectividad, motivaciones, estilo de vida e intereses. Todo esto asociado a la inexistencia de planes de tratamiento individuales integrales, canales establecidos de coordinación y comunicación insuficientes entre el equipo de trabajo y la ausencia de trabajo en equipo. Con este diagnóstico, se planteó la implementación del trabajo en equipo multidisciplinario con enfoque gerontológico, que desde el análisis retrospectivo, se identifican cambios significativos en la funcionalidad, calidad de vida y vinculación familiar de los adultos mayores, junto con mejorar el clima laboral y el autocuidado del trabajador.

La presente sistematización describe los modelos, etapas y metodologías desarrolladas durante un proceso de 7 años, con adultos mayores institucionalizados y de diferentes grados de funcionalidad; siendo los ejes centrales la articulación del equipo de trabajo, el manejo ambiental, la participación ocupacional, la vinculación familiar y la conexión con redes comunitarias.

El propósito de esta sistematización es destacar los aspectos determinantes del proceso de cambio del paradigma, desde una intervención biomédica geriátrica al de intervención gerontológica integral, motivando a profesionales y personas asociadas al trabajo con adultos mayores, para replicar y perfeccionar esta experiencia, que significó mantener la funcionalidad, el estado de salud general biopsicosocial y espiritual y potenciar el trabajo en equipo, disminuyendo la sobrecarga y optimizando funciones.

El principal objetivo es presentar estrategias de intervención con enfoque gerontológico que impactan en la mantención de la salud y la funcionalidad del adulto mayor institucionalizado; siendo 
un aporte para la toma de decisiones, no solo entre los equipos multidisciplinarios, sino también para aquellas personas vinculadas al trabajo con adultos mayores.

Por último, es importante señalar, que esta es la primera sistematización publicada del trabajo en equipo con enfoque gerontológico en nuestro país, ya que los esfuerzos se han orientado a la determinación del número de establecimientos de larga estadía a nivel nacional y regional. Un estudio de Marín, Guzmán y Araya (2004), citado en el Estudio de un Sistema y Plan de Mejoramiento de Calidad de Servicio que ofrecen los ELEAM (2007) de Asesorías para el Desarrollo; señala que no existe en Chile un registro fiable del número de establecimientos de larga estadía para el adulto mayor y de la población atendida por éstos. De acuerdo a una estimación estadística del mismo estudio antes citado, en la base de datos del Censo 2002, habrían en el país un total de 1.668 "hogares de ancianos", de los cuales $624(37,4 \%)$ serían "residencias colectivas informales" y $1.044(62,6 \%)$ "residencias colectivas formales". En la Región Metropolitana, según el mismo estudio, habría 804 residencias, divididas en 520 formales y 284 informales. Estos establecimientos atenderían a una población estimada de 14.178 adultos mayores. (1)

\section{DESCRIPCIÓN DEL DESARROLLO DE LA EXPERIENCIA}

El enfoque gerontológico permite abordar a las personas mayores desde una perspectiva multidimensional e integral para comprender sus cambios y necesidades en los aspectos biológico, médico, psicológico y social; pues estos influyen de manera directa en la forma como el ser humano asume su proceso de envejecimiento. Por otro lado, la gestión del cuidado se relaciona con el nivel de funcionalidad, participación, ejercicio de roles ocupacionales y vinculación con la familia; impactando la calidad de vida de los adultos mayores institucionalizados, percibido por el adulto mayor, su familia y el equipo.

El desafío de nuestro equipo multidisciplinario, fue lograr que cada integrante realizara su aporte especializado, considerando las relaciones y contribuciones de otras disciplinas. Luego, conocer e incorporar al trabajo diario, el enfoque gerontológico; condiciones necesarias para potenciar la efectividad del trabajo en equipo y el consiguiente impacto en el bienestar del adulto mayor. Teóricamente, y luego en la praxis, existió mayor disposición y compromiso de miembros del equipo ligados al trabajo psicosocial comunitario, no así en aquellos con un paradigma biomédico, entre los cuales se incluye algunos médicos, trabajadores de trato directo e incluso los propios adultos mayores y sus familias. Por ello, se hace necesario la socialización de este modelo, a través, de reuniones periódicas, jornadas de formación, análisis in situ de momentos críticos entre los integrantes del equipo y con los adultos mayores; promoviendo las relaciones horizontales, respetuosas y con responsabilidad en la toma de decisiones.

El proceso de incorporación de este nuevo paradigma de atención a los adultos mayores denominado Intervención con Enfoque Gerontológico, requirió la implementación de estrategias orientadas a incorporar este modelo en todos los ámbitos de atención del adulto mayor y su familia. Requirió además, la inducción en el modelo a cada persona que se incorporaba a la institución, cabe destacar que como estrategia fundamental se consideró el planteamiento explícito de la conformación del equipo, mencionando primero al Adulto Mayor y su familia y luego el resto de los integrantes (Médico Geriatra, Enfermera, Kinesiólogo, Terapeuta Ocupacional, Trabajadora Social y personal de trato directo).

Este proceso fue analizado y evaluado permanentemente, al inicio contrastando con la realidad vivenciada, en base a logros, aciertos y desaciertos y luego en base al cumplimiento de objetivos planteados en los planes de intervención. 


\section{MARCO TEÓRICO CONCEPTUAL}

Morris \& Tassara y Cols. (2007) mencionan que en España a partir de la Constitución de 1978, se impulsan de forma decidida políticas sociales, donde el Estado asume el papel de garante del bienestar de los ciudadanos. Dentro del marco constitucional, en el artículo 50, se encuentra el punto de partida del desarrollo de las políticas dirigidas a dar respuesta a las necesidades de las personas mayores. Como respuesta al mandato de dicho artículo, las 17 comunidades autónomas se dotaron de sus respectivas leyes de Acción Social y Servicios Sociales, a partir de las cuales se ha ido tejiendo un amplio sistema de atención social en general y especialmente de atención a los adultos mayores, que ha supuesto la potenciación de una red pública de servicios sociales comunitarios especializados. Posteriormente, en 1982, la Asamblea Mundial del Envejecimiento de Naciones Unidas, recomienda a los diferentes estados que desarrollen otro tipo de recursos y programas para dar respuesta a las necesidades sociosanitarias de los adultos mayores, que a la vez fueran útiles para alcanzar el objetivo del lema "envejecer en casa".

Según lo planteado por Corregidor (2010), en España, en el año 1991 se gesta el Plan Gerontológico Estatal 1992-1999 bajo la perspectiva "envejecer en casa". Desde ese momento y con el objetivo de dar respuesta a las preferencias manifestadas por las personas mayores en relación con permanecer en su entorno hasta que sea posible, se diseña una red de recursos sociales de apoyo domiciliario y comunitario para satisfacer estas demandas. La atención domiciliaria y la atención diurna son los recursos que más favorecen la permanencia de las personas mayores en el entorno habitual en el que desarrollan sus vidas. La institucionalización o ingreso en un centro residencial se convierte en la alternativa para aquellas personas mayores que no puedan o no deseen continuar en sus hogares. Los servicios sociales, al igual que los servicios hospitalarios se estructuran de forma escalonada para adaptarse a las necesidades sociales que puedan presentar las personas mayores. El servicio de ayuda a domicilio, los centros de día y los centros residenciales representan los tres recursos básicos de atención social en este país. (2)

Los Centros residenciales (ELEAM) para adultos mayores en España son un servicio más de los que existen dentro del conjunto de los recursos sociosanitarios, que en ocasiones se constituirán en un recurso para largas estancias o definitivo, y en otras darán respuestas temporales para intentar superar con éxito determinadas circunstancias que con cierta frecuencia rodean a los adultos mayores.... Estar adecuadamente ubicados en los entornos de procedencia de los adultos mayores, atender a un tamaño reducido de usuarios, contar en su plantilla con un equipo de profesionales con formación especializada en la atención de personas mayores, estar dotados de equipamientos básicos accesibles y tener una oferta amplia de servicios de ocio, rehabilitación y culturales (3)

En Chile las instituciones pioneras en atender las necesidades de las personas mayores fueron entidades privadas sin fines de lucro como el Hogar de Cristo, Fundación Las Rosas, Caritas-Chile, CONAPRAN, entre otras. Desde el año 1995, con la creación de una Comisión Ampliada, multisectorial y pluralista integrada por académicos, políticos, profesionales de los servicios públicos y de la sociedad civil, se comenzaron a desarrollar acciones emanadas desde el Estado para abordar la temática de adulto mayor en Chile. Esta comisión elaboro la Política Nacional del Adulto Mayor que fue promulgada en Marzo de 1996 cuya gran meta es lograr un cambio cultural en toda la población que signifique un mejor trato y valoración de los adultos mayores en nuestra sociedad, lo que implica una percepción diferente sobre el envejecimiento y alcanzar mejores niveles en su calidad de vida. La comisión se transformó en el Comité Nacional para el Adulto Mayor. Luego, en Septiembre de 2002, fue sustituida por el Servicio Nacional del Adulto Mayor (SENAMA) cuya misión es garantizar y promover el conocimiento y ejercicio de los derechos de las personas mayores, fomentar su plena integración a la sociedad y articular un sistema de protección social por medio de la coordinación, implementación y evaluación de políticas, planes y programas específicos. 
Nuestro país presenta una diversidad geográfica, cultural y económica, que influye en la distribución y densidad de la población. Según el estudio del SENAMA (2009) "Demanda de los Servicios de Cuidados para los Adultos Mayores", la territorialidad juega un papel primordial en la calidad de servicios de cuidado que un individuo puede esperar, dependiendo de su lugar de residencia. Además, las representaciones sociales que existen en nuestra sociedad sobre la vejez, donde la ancianidad se asocia a la presencia de enfermedades, a la falta de participación social y a la soledad; contribuyen a no visualizar la precaria y compleja realidad de los servicios de cuidado en nuestro país, a la cual normalmente es posible acceder en situaciones en que esta realidad nos aborda desde la experiencia individual o medios de comunicación que estigmatizan negativamente esta etapa del ciclo vital.

Dentro de los servicios de cuidado de adultos mayores, se encuentran los establecimientos de larga estadía, los cuales coexisten a nivel nacional con diferencias sustanciales en cuanto a cobertura, perfiles de usuarios, enfoque de intervención, dotación de personal, territorialidad, nivel de vinculación con la familia y la comunidad. Dado el aumento de este tipo de establecimientos, el año 2005 el Ministerio de Salud, en un intento por regularizar estas condiciones, modifica el reglamento, a través del Decreto $\mathrm{N}^{\circ} 134$, mencionando que "residen adultos mayores que, por motivos biológicos, psicológicos o sociales, requieren de un medio ambiente protegido y cuidados diferenciados para la mantención de su salud y funcionalidad, el cual cuenta con autorización para funcionar en esa calidad otorgada por la Secretaría Regional Ministerial de Salud competente del lugar en que se encuentra ubicado". El reglamento incluye disposiciones generales legales para la instalación y funcionamiento de la planta física, la dirección técnica, el personal y la fiscalización. Además plantea tanto para la dirección técnica como para el personal de atención de los Establecimientos de Larga Estadía para Adultos Mayores (ELEAM), como preferencia la capacitación en gerontología y en el articulo 19 menciona "es recomendable que estos establecimientos cuenten, además, con Enfermera, para la gestión de los cuidados, Nutricionista para la confección de minutas y dietas, Kinesiólogo, Terapeuta ocupacional o Profesor de educación física con formación gerontológica, para la rehabilitación y mantenimiento de las funciones biopsicosociales de los residentes".

Luego que un ELEAM ha cumplido todos los requisitos para comenzar a funcionar, el equipo interventor, integrado tanto por profesionales, técnicos y personal de trato directo; es primordial que puedan consensuar el paradigma, enfoque y modelos de intervención, los cuales deben estar en sintonía con el Plan Nacional del Adulto Mayor, el reglamento vigente y los conceptos actuales de envejecimiento activo, emanados de propuestas internacionales (ONU, CIPD, CORV) (4). Cabe mencionar que el año 2002 la OMS, propone el término Envejecimiento Activo como "el proceso de optimización de las oportunidades de salud, participación y seguridad con el fin de mejorar la calidad de vida a medida que las personas envejecen". Por ello, es relevante unificar criterios dentro del equipo interventor, no solo con la finalidad de dar cumplimiento a lo exigido por ley, sino también con el valor agregado de contribuir a la autonomía y al mejoramiento de la calidad de vida de las personas mayores y la construcción de una sociedad inclusiva.

\section{CONTEXTUALIZACIÓN}

El rápido envejecimiento de la población adulta mayor en América Latina y el Caribe, desde la década del '60, a la fecha, ha provocado cambios en la estructura de la pirámide demográfica de la región. Según proyecciones del CELADE (Centro Latinoamericano y Caribeño de Demografía), para el 2025 el porcentaje de adultos mayores alcanzará en promedio un 15\% y aumentará a 24,3\% el 2050 (5). Por ello, el año 1982 las Naciones Unidas presentó a sus miembros el Plan Internacional de Acción sobre el Envejecimiento, sin embargo, fue insuficiente para provocar un impacto significativo. No obstante, fue la base de la reflexión y desarrollo de propuestas de abordaje para esta etapa de la vida, claramente en aumento acelerado, que implica enfrentar nuevos desafíos a nivel individual, comunitario y político. En América Latina y El Caribe desde el año 1999 se han realizado asambleas, seminarios, conferencias y reuniones regionales que buscan la construcción de una sociedad basada en todas las edades con protección social basada en derechos. 
El Seminario Regional Técnico realizado el 2009, menciona que los diferentes Estados han implementado "reformas previsionales y del sector salud, en el funcionamiento de sistemas de protección social, en la institucionalidad pública y en la asignación de recursos" (6), incorporando acciones tendientes a cumplir las recomendaciones de la Declaración de Brasilia (2007), que señala "garantizar, promover y proteger los derechos humanos y las libertades fundamentales de todas las personas mayores, trabajar la erradicación de todas las formas de discriminación y violencia y crear redes de protección”. (7)

Todos estos encuentros regionales han permitido intercambiar experiencias, para materializar los compromisos acordados, buscando alternativas pertinentes a la realidad de cada país. Es así, como en Chile resulta vital tomar conciencia del cambio demográfico y sus características particulares en lo cultural, social, político y económico. Según la publicación del Servicio Nacional del Adulto Mayor (SENAMA) 2009, aspectos a considerar son:

- La mayor expectativa de vida de la población, debido a la reducción de la tasa de mortalidad, gracias al avance de la medicina y la implementación de políticas públicas, asociadas a la salud y el trabajo

- Cambios socioculturales, como el acceso de las mujeres a los medios de control de la natalidad y su creciente participación en el mercado laboral, lo que ha producido una reducción de las tasas de fecundidad. (8)

Estos dos fenómenos, explican la transición demográfica hacia el envejecimiento de la población, que en Chile se encuentra en etapa avanzada.

En los últimos 30 años, la población del país ha experimentado un proceso de envejecimiento demográfico acelerado y sin precedentes históricos. Hasta 1970, las personas mayores de 60 años representaban 8\% de la población, en el Censo de 2002 aumentaron a 11,4\% y en la Encuesta de Caracterización Socioeconómica (CASEN) 2006, las personas mayores de 60 años alcanzan el 13\% de la población total del país, es decir, 2.100 .378 personas. Para los próximos 20 años se estima una tasa de crecimiento de $3,7 \%$ anual para este grupo etario, por lo que se proyecta una población de 3.825 .000 personas de edad para el año 2025, lo que representará el $20 \%$ de los chilenos. (9)

El envejecimiento avanzado del país, se asocia principalmente al aumento de la esperanza de vida, que actualmente tiene un promedio de 78,6, siendo 81,5 para las mujeres y 75,6 para los hombres (10). Este nuevo contexto social implica oportunidades no solo para los adultos mayores de ser protagonistas en su proceso de envejecimiento activo, sino también para la sociedad al valorarlos como personas de derechos, participativas, capaces ejercer su autonomía personal; facilitando el intercambio intergeneracional para la construcción de una sociedad inclusiva donde todos tienen espacio para crear, aportar y adquirir nuevos roles ocupacionales, sin discriminación de ningún tipo.

Un indicador de salud y bienestar es la capacidad de la persona de adaptarse y participar en la sociedad. Las actividades de la vida diaria y los roles ocupacionales cumplen un papel fundamental en el autocuidado, la vinculación familiar y la participación social de los adultos mayores. La capacidad para realizar estas acciones permite a la persona mantener su autonomía y permanecer incluída en la comunidad, dentro de su entorno familiar y cumpliendo sus roles.

A nivel nacional se han realizado estudios, que muestran un porcentaje entre $21,4 \%$ y $25,5 \%$ de las personas de 60 años y más con dificultad en la realización de actividades de la vida diaria (11) y un 10,7\% discapacidad severa (12). Además, el Ministerio de Salud (MINSAL) estima un 3,3\% de prevalencia de personas mayores postradas. Estos antecedentes reflejan que uno de los principales desafíos del envejecimiento poblacional, es la dependencia, la cual genera un alto impacto tanto en la calidad de vida de las personas que la padecen como para quienes están a cargo de sus cuidados, así como altos costos para el sistema de protección social. Bajo este contexto, los profesionales y técnicos que trabajan directa o indirectamente en el área de geronto-geriatría, deben comprometerse con un nuevo enfoque de abordaje, de tipo socio sanitario, que incorpora la intervención psicosocial y 
comunitaria para mejorar la calidad de vida de las personas mayores. Muchas veces esta transición significa un desafío al trabajar con nuevos modelos y estrategias de intervención, que van desde el asistencialismo, el envejecimiento saludable propio del modelo biomédico hacia el actual concepto de envejecimiento activo y el enfoque de derechos; que incluye a los adultos mayores frágiles o en condición de dependencia.

Al revisar los principios en favor de las personas mayores propuestos el año 2009, como el reconocimiento de derechos, respeto a la integralidad y universalidad, valoración de la diversidad, envejecimiento en dignidad y democratización de los países (13); se puede postular que existe una brecha entre la situación de adultos mayores integrados en su familia y comunidad y aquellos que se encuentran institucionalizados ya sea por dependencia funcional, situación económica, familiar, social o por voluntad propia.

Considerando que nuestra sociedad, a pesar del envejecimiento avanzado, continúa entregando opciones de acceso a instituciones públicas y privadas que se limitan principalmente a intervenciones y tratamientos básicos del cuidado, en lugar de crear redes de servicio que den respuesta a las necesidades reales de las personas mayores, ya sea, al permanecer integrado en su familia, comunidad o en establecimientos de larga estadía. Por ello, se hace necesario sensibilizar a la población, especialmente a las personas relacionadas con la atención de personas mayores institucionalizadas, con la finalidad de incorporar esas intervenciones a un enfoque gerontológico de mayor pertinencia y atingencia a la situación individual.

\section{ESTRATEGIA METODOLOGICA}

Se utilizará el concepto de sistematización propuesto por el Instituto Interamericano de Derechos Humanos "registrar, de manera ordenada, una experiencia que deseamos compartir con los demás, combinando el quehacer con su sustento teórico, y con énfasis en la identificación de los aprendizajes alcanzados en dicha experiencia”.

Esta propuesta metodológica, rescata la experiencia de atención gerontológica en dos Hogares de una institución de beneficencia con vasta trayectoria en el tema; analizando las vivencias surgidas del quehacer diario y evidenciando cómo la práctica en sí, facilitó una conceptualización, estructuración y aprendizaje reflexivo del equipo multidisciplinario; permitiendo generar una propuesta metodológica coherente y desde nuestro punto de vista, con posibilidades de ser replicada.

Para determinar modelos de intervención idóneos en el trabajo gerontológico, fue necesario establecer criterios básicos comunes, fundamentándose inicialmente en modelos teóricos compartidos por las distintas disciplinas y en la selección de modelos teóricos de disciplinas específicas que se consideraron pertinentes de conocer e internalizar por todo el equipo. Para esquematizar la idea anterior, se plantea en la Figura $\mathrm{N}^{\circ} 1$, tres modelos de intervención que se utilizaron en la práctica y los aspectos más relevantes que cada miembro del equipo identificaba de ellos desde lo teórico (definición, valoración de sujeto) y su aplicación en lo cotidiano (campo relacional, cotidianeidad) (14):

Figura $\mathrm{N}^{\circ}$ 1: Esquema de Modelos de Intervención Gerontológica

\begin{tabular}{|c|c|c|c|}
\hline & Modelo de Autocuidado ${ }^{1}$ & Psicosocial & $\begin{array}{c}\text { MOH (Modelo de } \\
\text { Ocupación Humana) }\end{array}$ \\
\hline Definición & $\begin{array}{l}\text { Conjunto de intervenciones que ayudan al } \\
\text { individuo a llevar a cabo y mantener por } \\
\text { sí mismo acciones de autocuidado para } \\
\text { conservar la salud y la vida, recuperarse } \\
\text { de la enfermedad y/o afrontar las } \\
\text { consecuencias de dicha enfermedad }\end{array}$ & $\begin{array}{l}\text { Conjunto de intervenciones que } \\
\text { promueven la autonomía y el } \\
\text { funcionamiento de la persona en su } \\
\text { entorno, para lo cual, requiere } \\
\text { activar un sistema de servicios } \\
\text { comunitarios o soportes sociales } \\
\text { destinados a ofrecer los apoyos } \\
\text { necesarios }\end{array}$ & $\begin{array}{l}\text { Es una herramienta representativa, } \\
\text { derivada de la Teoría General de Sistemas } \\
\text { que explica la motivación hacia el alcance } \\
\text { de ocupaciones significativas y del cómo } \\
\text { el ambiente interactúa en el proceso }\end{array}$ \\
\hline
\end{tabular}

\footnotetext{
${ }^{1}$ Dorotea Orem, 1993

${ }^{2}$ Gary Kielhofner, 2002
} 


\begin{tabular}{|c|c|c|c|}
\hline $\begin{array}{l}\text { Valoración de } \\
\text { Sujeto }\end{array}$ & $\begin{array}{l}\text { Concibe al ser humano como un } \\
\text { organismo biológico, racional y pensante, } \\
\text { que recibe influencias del entorno. } \\
\text { Es una persona con capacidad de } \\
\text { conocerse, utilizar sus ideas, palabras y } \\
\text { símbolos para pensar, comunicar y } \\
\text { reflexionar, sobre su propia experiencia y } \\
\text { hechos colaterales, a fin de llevar a cabo } \\
\text { acciones de autocuidado. }\end{array}$ & $\begin{array}{l}\text { La consideración de sujeto se basa } \\
\text { en los derechos humanos y en la } \\
\text { valoración del campo de la relación, } \\
\text { para buscar autonomía e inclusión }\end{array}$ & $\begin{array}{l}\text { El Ser Humano es considerado como un } \\
\text { sistema abierto y dinámico que debe } \\
\text { reorganizarse para responder a las } \\
\text { demandas personales y del grupo social a } \\
\text { lo largo de la vida. } \\
\text { - El ambiente es la situación física, social } \\
\text { y cultural en la cual el sistema opera. } \\
\text { - El intake es el estímulo procedente del } \\
\text { ambiente (personas, objetos, eventos). } \\
\text { - El troughput es el proceso de análisis, } \\
\text { elaboración de esta información por parte } \\
\text { de nuestro organismo y la organización de } \\
\text { una conducta ocupacional. } \\
\text { - El output es el comportamiento } \\
\text { ocupacional. }\end{array}$ \\
\hline $\begin{array}{l}\text { Campo } \\
\text { relacional }\end{array}$ & $\begin{array}{l}\text { Se basa en la relación de ayuda y/o } \\
\text { suplencia, motivación y cambio del } \\
\text { comportamiento, considerando aspectos } \\
\text { como la percepción del problema, } \\
\text { capacidad de autocuidado, barreras o } \\
\text { factores que lo dificultan, recursos para el } \\
\text { autocuidado, etc. } \\
\text { La estrategia básica es la educación para } \\
\text { el autocuidado en las diversas condiciones } \\
\text { de salud/enfermedad, a través de: } \\
\text { 1.- Asistir } \\
\text { 2.- Orientar } \\
\text { 3.- Apoyar física y psicológicamente } \\
\text { 4.- Promover un entorno favorable al } \\
\text { desarrollo personal } \\
\text { 5.- Enseñar técnicas especificas }\end{array}$ & $\begin{array}{l}\text { Su premisa es que el campo } \\
\text { relacional influye en la construcción } \\
\text { del sujeto, ya que las interacciones } \\
\text { producen intercambio con otros } \\
\text { sujetos, por lo tanto, la persona es } \\
\text { porque las condiciones la producen. }\end{array}$ & $\begin{array}{l}\text { El ser humano organiza y ejecuta el } \\
\text { comportamiento ocupacional, a través de } 3 \\
\text { componentes: } \\
-\cdot \text { Volición (motivación): confianza } \\
\text { personal, valores e intereses } \\
\text { - Habituación: hábitos y rutinas } \\
\text { - Ejecución: habilidades }\end{array}$ \\
\hline Cotidianeidad & $\begin{array}{l}\text { El autocuidado es una actividad aprendida } \\
\text { por los individuos, orientada hacia un } \\
\text { objetivo. Es una conducta que existe en } \\
\text { situaciones concretas de la vida, dirigida } \\
\text { por las personas sobre sí mismas, hacia } \\
\text { los demás o hacia el entorno, para regular } \\
\text { los factores que afectan a su propio } \\
\text { desarrollo y funcionamiento en beneficio } \\
\text { de su vida, salud o bienestar. }\end{array}$ & $\begin{array}{l}\text { Se trabaja la intersubjetividad, los } \\
\text { significados, los sentidos y la } \\
\text { construcción simbólica; que } \\
\text { apunten hacia el desarrollo de } \\
\text { calidad de vida y bienestar del } \\
\text { adulto mayor en su entorno (familia, } \\
\text { comunidad, ELEAM) }\end{array}$ & $\begin{array}{l}\text { Las características y demandas del } \\
\text { ambiente condicionan las elecciones. A su } \\
\text { vez la elección es un acto volicional, } \\
\text { donde la confianza personal determina el } \\
\text { grado de desafío que un individuo espera } \\
\text { encontrar en el ambiente, la configuración } \\
\text { de valores e intereses determinan el tipo de } \\
\text { ambiente que será atractivo para la } \\
\text { persona }\end{array}$ \\
\hline
\end{tabular}

\section{ANALISIS DEL DESARROLLO DE LA EXPERIENCIA}

Teniendo en cuenta los modelos antes mencionados en la Figura $\mathrm{N}^{\circ} 1$, es posible definir una serie de etapas para el proceso de intervención por parte del equipo, dirigido a adultos mayores en ELEAM. La dinámica de un equipo gerontológico, implica la identificación de necesidades percibidas por el adulto mayor y su familia, siendo el punto de partida para el desarrollo de competencias por parte de los miembros del equipo, que potencian la mantención de la funcionalidad, participación social y autonomía.

Después de analizar la experiencia, se identifican una serie de aspectos claves en la dinámica de un equipo gerontológico que se grafica en la Figura $\mathrm{N}^{\circ} 2$, como un proceso continuo, activo, flexible, en constante evaluación, centrado en el adulto mayor y su familia, quienes influyen en forma permanente en cada una de las etapas planteadas:

Etapa 1: Conformación de equipo

Etapa 2: Definición de Planes de Intervención

Etapa 3: Difusión de los Planes de Intervención

Etapa 4: Ejecución de los Planes de Intervención

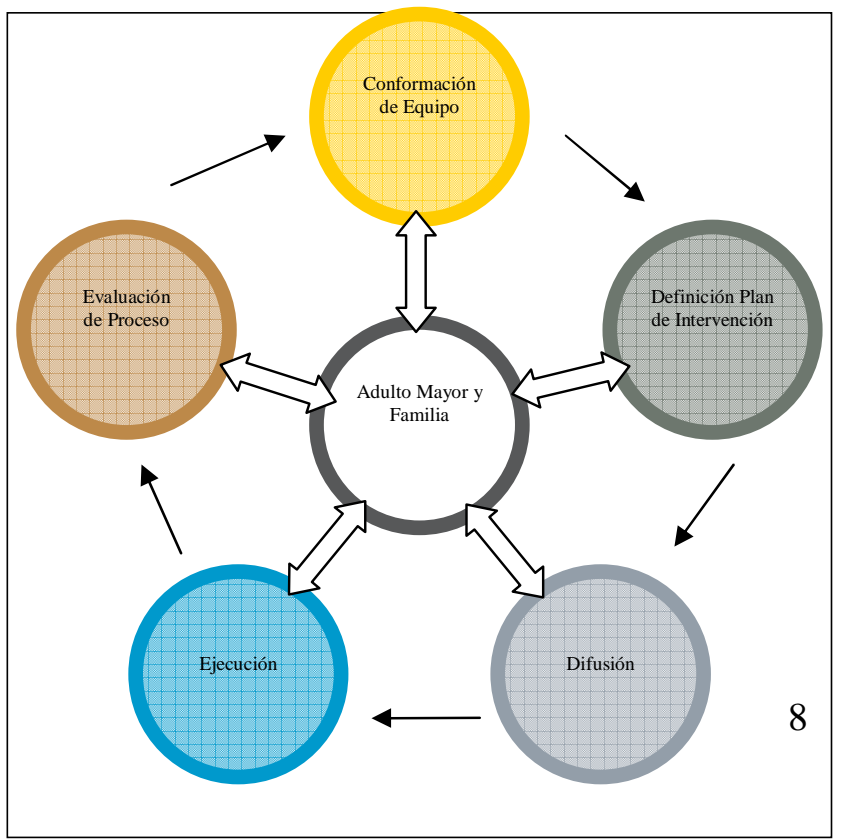


Etapa 5: Evaluación de proceso permanente

Figura $\mathrm{N}^{\circ}$ 2: Dinámica de un Equipo Gerontológico

Desde la experiencia vivida, estas cinco etapas deben ser identificadas como estratégicas, por lo cual se plantean acciones básicas indispensables en cada una, que se asocian a competencias a desarrollar por los miembros del equipo para la obtención de los resultados esperados, que en conjunto facilitan intervenciones con enfoque gerontológico.

En la Figura $\mathrm{N}^{\circ} 3$, se resumen las cinco etapas y las respectivas acciones, competencias y resultados, que se consideran básicas.

Figura $\mathrm{N}^{\circ}$ 3: Esquema de proceso de un Equipo Gerontológico

\begin{tabular}{|c|c|c|c|}
\hline ETAPAS & ACCIONES & COMPETENCIAS & RESULTADOS \\
\hline $\begin{array}{l}\text { Etapa 1: } \\
\text { Conformación de } \\
\text { Equipo }\end{array}$ & $\begin{array}{l}\text {-Unificar criterios } \\
\text {-Establecer roles y normas } \\
\text {-Reuniones de equipo }\end{array}$ & $\begin{array}{l}\text {-Dialogar } \\
\text {-Creatividad } \\
\text {-Organización }\end{array}$ & $\begin{array}{l}\text {-Trabajo en equipo } \\
\text {-Innovación }\end{array}$ \\
\hline $\begin{array}{l}\text { Etapa 2: } \\
\text { Definición Plan } \\
\text { de Intervención }\end{array}$ & $\begin{array}{l}\text {-Evaluación integral } \\
\text {-Identificación de necesidades, } \\
\text { pertinencia y contexto } \\
\text {-Incluir al AM y su familia }\end{array}$ & $\begin{array}{l}\text {-Integrar disciplinas } \\
\text {-Priorizar al AM } \\
\text {-Empatía }\end{array}$ & $\begin{array}{l}\text { amiento reflexivo } \\
\text { vención } \\
\text { ícativa para el } \mathrm{AM}\end{array}$ \\
\hline$\frac{\text { Etapa 3: }}{\text { Difusión }}$ & $\begin{array}{l}\text {-Informar acuerdos a los } \\
\text { actores involucrados } \\
\text {-Capacitación in situ }\end{array}$ & $\begin{array}{l}\text { - Entregar información } \\
\text { oportuna, clara } \\
\text { y precisa } \\
\text {-Comunicación }\end{array}$ & $\begin{array}{l}\text {-Compromiso del } \\
\text { equipo, el AM y la } \\
\text { familia }\end{array}$ \\
\hline$\frac{\text { Etapa 4: }}{\text { Ejecución }}$ & $\begin{array}{l}\text {-Cumplir acuerdos } \\
\text {-Colaboración } \\
\text {-Entrenamiento }\end{array}$ & $\begin{array}{l}\text {-Orientación al logro } \\
\text {-Calidad del trabajo } \\
\text {-Resolutividad }\end{array}$ & $\begin{array}{l}\text {-Funcionalidad } \\
\text {-Participación } \\
\text {-Autonomía del AM }\end{array}$ \\
\hline$\frac{\text { Etapa 5: }}{\text { Evaluación de Proceso }}$ & $\begin{array}{l}\text {-Supervisión } \\
\text {-Adaptación al cambio } \\
\text {-Definición áreas/procesos críticos }\end{array}$ & $\begin{array}{l}\text {-Retroalimentación } \\
\text {-Ética }\end{array}$ & lanteamiento oportuno \\
\hline
\end{tabular}

Etapa 1: Corresponde a la conformación del equipo, que también puede plantearse como estructuración o reestructuración del equipo según la situación contextual, en esta etapa se considera:

- La unificación de criterios como determinante y para ello se utilizó como base el esquema de modelos de intervención (figura $\mathrm{N}^{\circ} 1$ ) 
- El establecimiento de roles y normas, que considera la descripción de cargos, contrato laboral, normas laborales y técnicas nacionales e institucionales, y a la vez requerimientos internos del usuario y su familia

- Las reuniones de equipo, que constituyen un espacio de evaluación y retroalimentación relevante, deben tener una estructura y planificación, en ellas deben participar los miembros del equipo, el adulto mayor y su familia, según el objetivo y posibilidades de funcionamiento interno del ELEAM, la elaboración de calendario anual optimiza los tiempos de coordinación.

Las competencias a desarrollar por los miembros del equipo están asociadas al diálogo, la creatividad y la organización, esto implica motivación y compromiso personal, logrado en un contexto de clima laboral participativo. Los principales resultados obtenidos fueron el trabajo en equipo e innovación, existiendo una serie de iniciativas asociadas tanto a aspectos técnicos como de mejoramiento de la convivencia interna, por ejemplo: formulación de protocolos, sistemas de comunicación, formatos estadísticos, organización de grupos pastorales, actividades recreativas, socioculturales, optimización del aporte del voluntariado.

Etapa 2: Se asocia a la definición del plan de intervención y está centrada en el adulto mayor y su familia, considerando todos los ámbitos del ser humano. Este plan tiene como finalidad:

- Planificar y ejecutar las intervenciones

- Monitorear el progreso y evaluar la eficacia de las intervenciones

- Facilitar la comunicación y coordinación de las intervenciones entre los miembros del equipo

Es importante mencionar que durante la experiencia de trabajo, el equipo planteó un formato inicial de plan de intervención, dividiendo las evaluaciones individuales por disciplina, el cual se fue perfeccionando, llegando a un formato integral, en el cual se identifican problemáticas, objetivos y actividades de manera conjunta y se asignan responsabilidades en base al contexto y la experticia de cada uno, teniendo presente los modelos de intervención de la Figura $\mathrm{N}^{\mathrm{o}}$ 1. Este plan de intervención debe formularse en instancias formales como reuniones de equipo calendarizadas y estructuradas (definición de tabla, horarios, participantes), a la vez ser reevaluado periódicamente. El pensamiento reflexivo como resultado vivenciado, permite mejorar la capacidad del equipo para identificar intervenciones que para el adulto mayor y su familia son significativas.

Etapa 3: La difusión de los planes de intervención, facilitan el compromiso con el plan de intervención por parte del equipo, el adulto mayor y su familia. Se basa en la comunicación efectiva, a través de la entrega de información a las personas involucradas, que puede ser verbal o escrita, formal o informal, lo importante es que los canales de comunicación sean explicitados como tales. En esta experiencia se identifica la capacitación in situ como una acción habitual y que por un tiempo fue subvalorada, pero al analizar el impacto observado se planteó como parte de los programas de capacitación del ELEAM, con una estructura general y definiendo temática, objetivos y atribuciones de los responsables de ejecutarlas.

Etapa 4: Respecto a la ejecución de los planes de intervención, la experiencia nos mostró que los resultados de funcionalidad, participación y autonomía de los adultos mayores, dependen del resultado de las etapas anteriores, pues si no hay compromiso, pensamiento reflexivo del equipo, planteamiento de intervenciones significativas, trabajo en equipo e innovación; se ejecutan planes de intervención centrados en los intereses del equipo, con orientación al logro personal, sobre intervenciones, incumplimiento de compromisos y principalmente con enfoque biomédico. Desde la vivencia, el enfoque gerontológico se logra en un proceso conjunto de reflexión y diálogo para lograr un cambio de paradigma, a la vez con disposición a desarrollar o mejorar algunas competencias, lo cual requiere de apoyo mutuo por parte del equipo.

Etapa 5: Se tiende a considerar la evaluación como una etapa final, pero en este caso y como se observa en las Figuras $\mathrm{N}^{\circ} 2$ y 3 , se plantea como evaluación permanente de cada etapa y del proceso que cada una tiene. Para ello, las acciones de supervisión, adaptación al cambio y definición de áreas y/o procesos críticos, se consideraron relevantes. La supervisión ejercida por los distintos miembros del equipo, según el contexto, concebida como una responsabilidad de acompañamiento, contención, 
diálogo, enseñanza-aprendizaje, capacitación in situ; permite la monitorización y mejoramiento del plan de intervención. Acá es relevante la adaptación al cambio y en la práctica ha sido efectivo la utilización de los modelos planteados en la Figura $\mathrm{N}^{\mathrm{o}} 1$ y la intervención centrada en el usuario y su familia, para evitar la frustración de los integrantes del equipo.

\section{CONCLUSIONES}

Las estrategias para incorporar esta forma de trabajo en los equipos son diversas y dependen de la realidad local de cada establecimiento, sin embargo, existen estrategias básicas aplicables, las cuales se plantean a continuación:

1) Visita del AM y familia previa al ingreso. La finalidad de este primer acercamiento es que el AM y la familia conozcan el entorno, infraestructura y funcionamiento interno del establecimiento (horarios, normas, factores protectores y de riesgo, opciones de participación y elección ocupacional). Esta estrategia basada en los principios éticos de beneficencia, no maleficencia, autonomía y justicia, permite el consentimiento informado, la credibilidad y confianza en el equipo interventor (que considera actores claves al AM y su familia).

2) Establecer procedimientos y protocolos de comunicación interna, mecanismos necesarios que potencian la gestión del cuidado integral en los distintos niveles de intervención: individual, grupal, social tanto de usuarios como trabajadores. Las formalidad básicas que debieran definirse e implementarse corresponden a procedimientos/protocolos de: ingreso, egreso, gestión del cuidado, urgencias, estimulación/entrenamiento, vinculación familiar, participación comunitaria, traslados, uso del tiempo libre, manejo ambiental, entre otras

3) Descripción de cargo. Es un proceso en el cual se realiza un listado detallado con los aspectos significativos del cargo, sus deberes y responsabilidades, que facilitan el establecimiento de las relaciones dentro del grupo de trabajadores, entre pares y superiores; disminuyendo así los posibles conflictos al ejercer las atribuciones pertinentes a ese cargo.

4) Liderazgo democrático y participativo. Proceso de influír en otras personas y motivarlas a participar y trabajar entusiastamente hacia el cumplimiento de un objetivo en común. Fomenta la discusión del grupo y considera las opiniones de todos sus integrantes. Las normas y criterios de funcionamiento son explícitos y claros, mejorando así el clima laboral y el autocuidado. Finalmente, esto se traduce en una atención ética, de calidad y centrada en el usuario.

5) Calendarizar reuniones formales de coordinación de equipo, con familiares y redes comunitarias. Es necesario la realización de reuniones periódicas con todos los actores involucrados, a modo de favorecer la evaluación de proceso y con ello, planificar y organizar oportunamente. Cabe mencionar, que esta estrategia contribuye a mejorar la comunicación, el sentido de pertenencia y la articulación con redes comunitarias.

6) Capacitación continua de profesionales y técnicos. Deben basarse en las necesidades emergentes del trabajo diario y las motivaciones del personal, desarrollando un pensamiento reflexivo, mejoramiento de los resultados y validando la autorrealización. Los trabajadores, sin importar el cargo, deben sentir que son un aporte real en la calidad de vida de los adultos mayores, que debiera trascender el ámbito laboral. Hoy en día, la estrategia de coaching da apoyo, facilita, educa y permite retroalimentación sobre desempeño y desarrollo de competencias personales y profesionales.

7) Capacitación continua del adulto mayor y su familia. El adulto mayor conserva su potencial de aprendizaje y autocuidado, y de acuerdo a teorías psicológicas, el individuo que envejece, está 
siempre aprendiendo, adaptándose y desarrollándose ${ }^{3}$. En esta mirada, es imprescindible buscar instancias de aprendizajes significativos, con sentido y trascendencia para validar al adulto mayor como actor principal de su envejecimiento activo. Un papel relevante cumple también la familia, a quienes se debe involucrar en el enfoque gerontológico, abordando inicialmente, sus necesidades inmediatas y luego continuar el proceso de mirada gerontológica.

8) Participación ocupacional del adulto mayor: Kielhofner (2002) define la participación ocupacional como la "involucración en actividades productivas, juego o del diario vivir que son parte del contexto sociocultural y son necesarias para el propio bienestar". En esta definición se manifiesta que las actividades que la persona mayor realiza están directamente relacionadas con su historia de vida, intereses, motivaciones y contexto. Se realizan, además, en diferentes áreas del desempeño ocupacional y que al adulto mayor corresponderían las siguientes:

a) Autocuidado: El año 2008 la American Occupational Therapy Association (AOTA) definió las actividades básicas de la vida diaria (AVDB) como "las actividades encaminadas al cuidado de nuestro propio cuerpo y que se encuentran relacionadas con la supervivencia del individuo", refiriéndose al desempeño funcional y lo más independiente posible de todas aquellas actividades de automantención, como por ejemplo, alimentarse, hidratarse, desplazarse, regulación del sueño, control de esfínteres, autocuidado, higiene y arreglo personal, actividad sexual y cuidado de ayudas técnicas. Por su parte, las actividades instrumentales de la vida diaria (AVDI) fueron definidas como "actividades orientadas a la interacción con el medio, son a menudo complejas y generalmente opcionales para hacer (se pueden delegar en otros)", contemplando: cuidado de otros, uso de sistemas de comunicación, desplazamiento en la comunidad, manejo de temas financieros, cuidado de salud y manutención, mantención del hogar, preparación de los alimentos, limpieza de los espacios, procedimientos de seguridad y respuestas de emergencia.

b) Productivas: referidas a participación en cursos, voluntariado, charlas, reuniones, trabajos informales, trabajos colaborativos, ser integrante de una organización, planificación de la jubilación.

c) Tiempo libre: valida el uso del tiempo en actividades de ocio, recreación y descanso.

Todas estas actividades deben estar contextualizadas en un ambiente significativo, facilitador de procesos y oportunidades de interacción, en el cual no existan restricciones (horarios de visita, acceso a información), limitaciones (acompañamiento en el traslado interno o externo, actividades sociorecreativas en la comunidad, trámites) o resistencias a la participación (desvalorización de potencialidades para el uso de servicios comunitarios -iglesia, feria, derecho a sufragar-). Un adulto mayor que participa de sus ocupaciones, adquiere un nivel de funcionalidad que se traduce en bienestar y autonomía.

Nos parece importante mencionar programas basados en la participación ocupacional individual o grupal:

* Entrenamiento específico: motor (caminatas asistidas, kinesiterapia motora, activación física, baile entretenido), cognitivo (lectoescritura, literatura, orientación temporoespacial, cine/TV) AVDB (educación de autocuidado, rutinas significativas), AVDI (manejo del dinero, ingesta de medicamentos, alimentación saludable, uso del teléfono y medios de comunicación, repostería/amasandería)

* Roles ocupacionales: programas de empleabilidad interna con proyección a la inserción laboral externa (vendedor de kiosko, portería, junior, carpintería, electricidad, auxiliar de aseo y/o cocina y cualquier otro oficio/profesión), voluntariado, talleres (reciclaje, jardinería, cocina)

* Actividades recreativas: Dentro y fuera del establecimiento, atingentes al contexto sociohistórico nacional y personal (cumpleaños, Fiestas patrias, Navidad, Año nuevo, día del adulto mayor, celebraciones, eventos, deportes, juegos de salón)

\footnotetext{
${ }^{3}$ Principio Epigenetico, Erickson, 1959.
} 
* Participación comunitaria: Acceso a la organización y manifestación grupal (asambleas internas, asociatividad en clubes). También el ejercicio y respeto por los derechos (servicios de salud, trámites legales, municipales, previsionales u otros, actividades espirituales, participación en organizaciones sociales, salidas propuestas por el AM -cementerio, mall, compras-)

* Vinculación familiar: Contacto telefónico, visitas del adulto mayor a la familia, horario flexible para las visitas familiares, fomento de actividades conjuntas.

9) Manejo ambiental. La mantención de un ambiente facilitador debe proveer un lugar seguro y estimulante para todos los integrantes del equipo (AM, familia, profesionales, técnicos, personal de trato directo y voluntarios). El concepto de manejo ambiental es clave a la hora de implementar programas, planes de intervención y actividades de vinculación con la familia y comunidad, que debe considerar los siguientes aspectos, propuestos por Kielhofner (2002):

a) Disposición del ambiente físico: Objetos disponibles, flexibles en el uso y variedad, complejidad atingente (conocidos, simples, atractivos) y significado (valor personal en el pasado y presente). Todas las modificaciones al ambiente físico deben ser socializadas y difundidas a todos los integrantes del equipo.

b) Actividades atractivas y significativas. Se relacionan con la motivación del adulto mayor para facilitar la participación ocupacional. Por ello, al momento de planificar y proponer actividades, es necesario considerar, el nivel de complejidad de la tarea, su temporalidad, las reglas o estructuras, la claridad del objetivo (seriedad o diversión) y la dimensión social (individual o grupal)

c) Grupo social y la relación con la persona: El aspecto social es el más importante para desarrollar confianza y empatía, donde la comunicación es determinante, ya que las palabras o gestos pueden entregar un mensaje confuso y crear sentimientos negativos. El grupo social debe tener un tamaño adecuado, objetivos y metas comunes, ser permeables al ingreso/egreso de otras personas y claridad de límites. En los ELEAM existe un gran número de personas, con diversidad cultural, por lo cual, la convivencia puede ser un factor desencadenante de conflictos entre pares y con el equipo. Frente a esto, el manejo ambiental del grupo, debe buscar la identificación de pertenencia a un grupo social específico, en forma voluntaria, según afinidad, para la participación ocupacional satisfactoria.

d) Activación de redes comunitarias: Es prioritario que el equipo cuente con catastro actualizado de las redes comunitarias territoriales, además de estar coordinados con los principales actores de esta red. La relación no debe estar basada en el "utilitarismo", sino que en la asociatividad y colaboración mutua, enfocados en el bienestar de los adultos mayores del ELEAM, pues ellos también son parte de la red comunitaria.

Con el planteamiento de estas estrategias, basadas en nuestra experiencia, como aporte metodológico, pretendemos sensibilizar a profesionales y personas relacionadas con adultos mayores, respecto a la relevancia y factibilidad de un cambio de paradigma desde intervenciones con enfoque biomédico a intervenciones con enfoque gerontológico.

El desconocimiento de los beneficios asociados a este planteamiento de intervención, predispone al prejuicio de aumento en los costos económicos, principalmente relacionados con profesionales formados con un enfoque gerontológico y la posterior capacitación del personal, sumado a la inversión en insumos y/o servicios de los diferentes programas; perpetuando así la atención geriátrica con enfoque biomédico. En nuestra experiencia, consideramos que obtuvimos resultados de costo/beneficio eficientes y eficaces, primero para el equipo en aspectos administrativos de gestión, cohesión del equipo, satisfacción con el trabajo, clima laboral que reduce ausentismo laboral, accidentes laborales y rotación de personal. Asimismo, nuestra propuesta de intervención, optimizó los resultados en cuanto a la funcionalidad en el adulto mayor y su familia. 
La institucionalización de las personas mayores impacta física, cognitiva y socialmente, además de la participación ocupacional, y se extiende a su grupo familiar, quienes muchas veces no cuentan con herramientas para manejar esta situación, incluso la posibilidad de institucionalización transitoria, es un concepto que no está internalizado en el imaginario colectivo. En las personas mayores que no cuentan con otra opción a la institucionalización, la atención gerontológica apropiada, puede incidir en las expectativa de vida y bienestar, es decir, es posible mantener la funcionalidad y retardar/controlar el deterioro asociado a las complicaciones de enfermedades crónicas.

Otro aspecto importante es el mejoramiento en la calidad de atención, que si bien existe la intención de certificar a los ELEAM, aun no se concreta y solo se realizan fiscalizaciones aleatorias. No obstante, a mediano plazo, al igual que en España y países desarrollados, es posible que el concepto de certificación de calidad para los ELEAM se instale en nuestro país, requiriendo en ese momento reestructuración radical del enfoque de intervención. La atención de calidad considera además, el trato digno, cálido y personalizado, valorando al adulto mayor como sujeto de derechos, conceptos integrados en el enfoque gerontológico.

En los equipos de intervención, es necesario la disposición al cambio de paradigma, estilos de trabajo y desarrollo de competencias; focalizando la toma de decisiones centradas en el individuo y en los principios bioéticos. Otra consideración innovadora es contemplar que dentro del equipo, también está el adulto mayor y su familia como actores más dentro de las distintas etapas del proceso, lo que requiere de un cambio, desde una actitud pasiva a un rol de protagonista en el proceso. Todos los integrantes pueden aportar, modificar, mejorar o replantear la ejecución del plan de intervención durante el proceso, gracias al compromiso del equipo en el logro de la autonomía.

Al realizar esta sistematización, evidenciamos que el aprendizaje obtenido en la práctica, puede replicarse y es necesario profundizarlo en un estudio de investigación y así, aportar al mejoramiento de la calidad de vida de los adultos mayores en establecimientos de larga estadía.

\section{BIBLIOGRAFIA}

(1) MARIN P., GUZMÁN J.M. y ARAYA, A. Adultos Mayores Institucionalizados en Chile: ¿Cómo Saber Cuántos Son? Revista Médica de Chile 2004; 132 (7): 832-838.

(2) (3) CORREGIDOR A. Terapia Ocupacional en los Servicios Sociales Comunitarios: servicios de ayuda a domicilio, centros de día y residencias. En: Terapia Ocupacional en Geriatría y Gerontología. Madrid, España: Editorial Ergon, 2010; 105 - 118.

(4) CORV (Coordinación de Organismos Regionales de la Sociedad Civil de América Latina y El Caribe). Envejecer con Derechos "Principios y Estrategias de la Sociedad Civil de América Latina y El Caribe”. Chile, Octubre 2009

(5) (6) Avances y Acciones Clave para la Implementación del Programa de Acción de El Cairo”, 6 y 7 Octubre 2009. Análisis de los avances en la implementación en América Latina del Programa de Acción (PA) de la Conferencia Internacional sobre la Población y el Desarrollo (CIPD).

(7) Foro Regional sobre Envejecimiento de Organizaciones de la Sociedad Civil de América Latina y El Caribe. Diciembre 2007

(8) (9) SENAMA. Las Personas Mayores en Chile: Situación, Avances y Desafíos del Envejecimiento y la Vejez. Julio 2009. 
(10) Boletín Demográfico 2007. Datos del documento "World Population Ageing, División de población de Naciones Unidas, 2007

(11) Albala C, García C, Lera L. Encuesta sobre Salud, Bienestar y Envejecimiento en Santiago, Chile. Estudio SABE. Santiago; 2007.

(12) FONADIS, Primer Estudio Nacional de la Discapacidad en Chile. Santiago Chile, 2004.

(13) Envejecer con Derechos. Principios y Estrategias de la Sociedad Civil de América Latina y El Caribe. Coordinación de Organismos Regionales de la Sociedad Civil de América Latina y El Caribe, Sede CEPAL, Chile, Octubre 2009.

(14) VÉLIZ V, URIBE L. Aportes de la Terapia Ocupacional al Contexto Educacional Inclusivo: Interrelación entre el Enfoque Psicosocial, la Teoría de Integración Sensorial y Acciones de Atención Temprana. Revista Chilena de Terapia Ocupacional 2009; 9: 109 